\title{
Vulnerable Patients and Potential Harms: The Contribution of Observational Research
}

\author{
Deborah Layton ${ }^{1}$
}

Published online: 28 January 2016

(c) Springer International Publishing Switzerland 2016

In this edition of Drug Safety, Bloechliger et al. present the results of their pharmacoepidemiological study that describes an evaluation of the risk of seizures associated with incident antidepressant use at usual therapeutic doses in patients with depressive disorder [1]. The study applies a case-control design nested within a cohort of patients with the full spectrum (mild-severe) of depressive disorders requiring treatment in the UK Clinical Practice Research Datalink (CPRD). Importantly, patients diagnosed with epilepsy and/or treated with antiepileptic medications, and/ or with important risk factors for seizures prior to the date of their first antidepressant prescription, were excluded in order to minimise confounding by pre-existing diagnosed diseases associated with seizures. In addition, patients with mild or transient depression not requiring treatment have also been excluded. The study not only suggested a difference in crude incidence rates of first-time seizure between subtypes of antidepressants (therapeutic subclass and generation) but also shows that early use may be important. With regard to monotherapy users of the newer antidepressants [selective serotonin reuptake inhibitors (SSRIs) and related serotonin noradrenaline reuptake inhibitors (SNRIs)], during the observation period the crude incidence rates per 10,000 person-years were 12.44 [95\% confidence interval (CI) 10.67-14.21] in SSRI users and 15.44 (95 \% CI 8.99-21.89) in SNRI users. The corresponding crude incidence rates appeared slightly lower for tricyclic antidepressant (TCA) current monotherapy

Deborah Layton

deborah.layton@dsru.org

1 Drug Safety Research Unit, Bursledon Hall, Blundell Lane, Southampton SO31 1AA, UK users and depressed, untreated patients $(8.33,95 \% \mathrm{CI}$ 4.68-11.98; and 9.33, $95 \%$ CI 6.19-12.46, respectively). With regard to timing, the authors note that crude incidence rates were highest shortly after the start of any monotherapy treatment $(12.58,95 \%$ CI $11.03-14.13)$, but lower in those regarded as past users $(5.05,95 \%$ CI 4.49-5.62). The case-control analysis, which provided adjusted stratified estimates of relative magnitude of risk of seizure according to current or past use compared with non-use, supported this pattern, with a doubling of incident seizure risk in current users of SSRIs and SNRIs, but not TCAs (adjusted odds ratio 0.99, $95 \%$ CI $0.63-1.53$ ), compared with non-users. No such difference was observed for past use. Furthermore, in the case-control analysis, when data were stratified by sex, adjusted odds ratios were also elevated in current users of SSRIs and SNRIs compared with non-users. An indication of a positive dose response relationship for some individual antidepressants (venlafaxine and mirtazapine) was observed.

This study raises a number of interesting points and provides additional important information about known risks, particularly in vulnerable populations. In the UK, antidepressants are one of the most commonly prescribed drugs in the primary care setting, managed primarily by general practitioners (GPs) [2]. They are not only used for the treatment of depression but also several other neuropsychiatric disorders. Several subtypes of antidepressants are available that exploit particular effects on selected neurotransmitter systems to achieve beneficial therapeutic outcomes; however, the wide use of antidepressants comes with corresponding health risks, as highlighted by this study.

The origin of the reported association stemmed principally from randomised controlled trials (RCTs), and 
informed on risk associated with high dose use, as well as untreated depression. That RCTs generally poorly reflect real-life situations is well known, as are their limitations in studying vulnerable groups such as the elderly or those with concurrent illnesses who have physical or cognitive impairments [3]. The study of safety issues associated with medicines in clinical practice can be better supported through conducting good-quality observational research. Bloechliger et al. chose to address this particular research question using a primary care electronic health records (EHRs) database availability of EHRs has made it possible not only to conduct observational studies of such associations in very large cohorts but also to contextualise results with other treatments and evaluate the appropriateness of prescribing [4]. EHR databases offer uniformity of data, with processes in place to addressing concerns regarding data privacy [5]. Use has been embraced by regulators, marketing authorisation holders and researchers alike in providing real-world data. However, despite the application of sophisticated analytical tools and methods to extract and analyse data, challenges remain in repurposing data for studying the safety of medicines. Generally, data in administrative databases record health information as a byproduct of financial transactions, whilst data in medical records databases are recorded as part of the process of clinical outpatient care and can be enhanced by linkage to other data sources, e.g mortality statistics data. Neither of these two types of databases are compiled with observational research as the primary objective. This particular study by Bloechliger et al. highlights the complexity of extracting the relevant data, which may require the generation of complex product and disease code lists. In addition, many assumptions must be made regarding relationships between diagnoses and treatment, and complex algorithms may be required to define certain metrics such as dose or duration of exposure, some of which also require assumptions. Misclassification and confounding by indication are real threats to study validity if these assumptions are misplaced. Such assumptions not only require expert knowledge of the database architecture but also an understanding of the health service that the data reflect and knowledge of the clinical research question.

Nevertheless, the importance of observational studies and the utility of EHRs in identifying vulnerable subpopulations at risk is demonstrated by Bloechliger et al. For example, by excluding patients diagnosed with depression and a prior history of epilepsy, there exists the possibility of a population with depression with a concurrent underlying but undiagnosed seizure disorder who are susceptible to seizures under certain conditions which may lower the seizure threshold, such as with the initiation of antidepressants [6]. Since incidence rates appeared to be lower in past-users than non-users, the authors identified that there exists a population with untreated depression who are also at risk of seizures [7]. In this study, the authors make no clear distinction between the type or nature of seizure in their evaluation; however, it is plausible that the seizures subsequently observed are most likely to be symptomatic or dissociative seizures rather than associated with a diagnosis of epilepsy [7]. This study has also identified a large group of past users. It is not known why they stopped treatment, but the observation that risk is higher in current users than past users is also consistent with the phenomenon of depletion of susceptibles (where patients who were susceptible for a seizure after starting antidepressant therapy developed the seizure early on, while longer term users were less susceptible) [8].

Importantly, Bloechliger et al. also explored the effect of antidepressant use in patients with pre-existing dementia, another important vulnerable group. In this study, because matching of cases with controls included age, it was only realistically possible to elucidate age-dependent patterns of risk from the reported crude incidence rates, which suggests that risk appears elevated in the older age group for current monotherapy SSRIs or SNRIs, but not TCAs. The interesting finding from a prespecified subgroup analysis from this study was the sixfold increased risk of incident seizures in depressed patients with preexisting dementia who were current users of SSRIs compared with non-users (adjusted odds ratio 6.74, 1.53-29.62), although this is based on fairly small numbers. This relationship in the SNRI and TCA drug classes for this subpopulation could not be examined because their use was low, which is not unexpected given recommendations to avoid antidepressant drugs with anticholinergic effects because of their adverse effect on cognition [9]. The corresponding analysis in patients without dementia was less remarkable.

With regard to studying risk of seizure in this vulnerable population with depression and dementia, a number of challenges ensue that may lead to criticism in the robustness of any observational study conducted within an EHR database. For example, the size of the eligible sample that can be studied may be low due to the inverse association of depression with age, despite a positive association between dementia and age. There may also be underreporting of depressive symptoms (either by the patient or their carer) to the physician, and/or problems in diagnosis of either depression or dementia due to frailty, multiple medication use, variation in presentation of psychiatric symptoms and criteria used to diagnose neuropsychiatric conditions. Although the association between depression and dementia is well known, the causal relationship remains unclear. A recent meta-analysis reported that depression was an independent risk factor for dementia [10]; however, recent cognitive neuroscience research suggests this association is 
independent of neuropathological hallmarks of dementia [11]. Epileptic seizures also occur in patients with dementia (particularly Alzheimer's disease) at a higher prevalence than among healthy elderly individuals [12]. Because manifestations of seizure may be misclassified as symptoms of underlying dementia, it may be possible that the observed seizure risk in depressed patients with dementia is even higher than that reported. Nevertheless, there is no reason to believe that this would have a differential effect across antidepressant classes if all were prescribed equally to this population. However, because prescribing recommendations advocate the use of SSRIs, it is not unexpected that risk appears highest in this group because there is no corresponding analysis in the other groups with which to contextualise findings.

What is known is that the prevalence of depression and dementia is higher within institutional facilities than within the community setting; thus, antidepressant use is likely to be correspondingly more frequent [13]. If the findings by Bloechliger et al. are taken in conjunction with other similar research supporting that antidepressants increase the risk of seizure, there exists a real possibility of exposing vulnerable patients to additional medication-related risks. Indeed, a report published in October 2015 by the UK Care Quality Commission highlighted the ongoing problems around poor medicines management in care homes in general [14]. Herewith is another limitation of the use of EHRs, in that whilst information on clinical practice within the primary care setting may be available, the ability to study the use of medicines in populations cared for within UK institutional facilities is partly dependent on whether responsibility for prescribing falls within or between secondary or primary care health services and where such information gets recorded. Only summary information from secondary care is available within primary care records although enhancements through linkages to other data sources are possible. Thus, new research initiatives are required in the UK to address this gap.

Finally, a brief comment about the research study design itself. The nested case-control (NCC) study design was first described in 1973 within the context of occupational epidemiology studies [15]. Over the years, the design was extended and refined, and the methodology was demonstrated to produce estimates of risk that were similar to the relative hazard in a proportional hazards model of time to response [16]. In contrast to a traditional case-control study, an NCC design is 'nested' in a cohort, and cases and controls represent random samples within that same study base [17]. The NCC design is an efficient approach that is particularly useful in circumstances where analysing the entire cohort adds little scientific value to addressing the research question albeit modern day computing power has improved analytical efficiency of large cohorts. Despite the

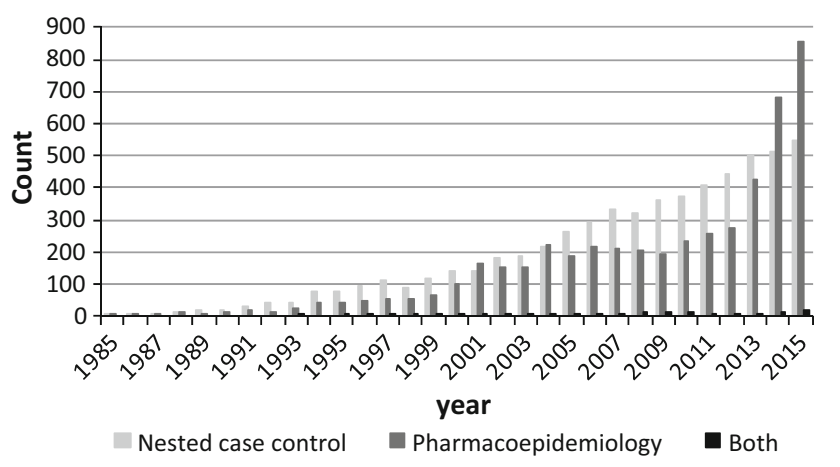

Fig. 1 MEDLINE publication counts between 1985 and 2015, using the indexed MeSH terms 'nested case control' and 'pharmacoepidemiology', as well as the intersection of both these terms. $\mathrm{MeSH}$ Medical Subject Heading

potential for research, a simple evaluation of MEDLINE publications between 1993 and 2015 based on the key Medical Subject Heading (MeSH) terms 'nested casecontrol' and 'pharmacoepidemiology' suggests, albeit crudely, that while the overall frequency of publications mapped to NCC study design $(n=5984)$, and similarly to pharmacoepidemiology $(n=4945)$, has increased, such growth has not been observed in the corresponding number of NCC studies linked specifically to pharmacopeidemiological research $(n=141)$ (Fig. 1). Is this observation merely an artefact of indexing or does it indicate the NCC has never really achieved favour within the pharmacoepidemiological research community? I hope it is the former.

\section{Compliance with Ethical Standards}

Conflicts of interest Deborah Layton is an employee of the Drug Safety Research Unit (DSRU). The Unit is an independent charity (No. 327206), which works in association with the University of Portsmouth. It receives unconditional donations from pharmaceutical companies. The companies have no control on the conduct or the publication of studies conducted by the DSRU.

Funding No sources of funding were used in the preparation of this editorial.

\section{References}

1. Bloechliger M, Ceschi A, Ruegg S, Kupferschmidt H, Kraehenbuehl S, Jick SS, et al. Risk of seizures associated with antidepressant use in patients with depressive disorder: follow-up study with a nested case-control analysis using the Clinical Practice Research Datalink. Drug Saf. 2015. doi:10.1007/s40264-0150363-z (Epub 9 Dec 2015).

2. Harris T, Carey IM, Shah SM, DeWilde S, Cook DG. Antidepressant prescribing in older primary care patients in community and care home settings in England and Wales. J Am Med Dir Assoc. 2012;13(1):41-7.

3. European Medicines Agency. ICH Topic E7. Studies in support of special populations: geriatrics. Available at: http://www.ema. europa.eu/docs/en_GB/document_library/Scientific_guideline/ 2009/09/WC500002875.pdf. Accessed 20 Dec 2015. 
4. Abbing-Karahagopian V, Kurz X, de Vries F, van Staa TP, Alvarez Y, Hesse U, et al. Bridging differences in outcomes of pharmacoepidemiological studies: design and first results of the PROTECT project. Curr Clin Pharmacol. 2014;9(2):130-8.

5. Andrews EB, Margulis AV, Tennis P, West SL. Opportunities and challenges in using epidemiological methods to monitor drug safety in the era of large automated health databases. Curr Epidmiolol Rep. 2015;1:194-205.

6. Cardamone L, Salzberg MR, O'Brien TJ, Jones NC. Antidepressant therapy in epilepsy: can treating the comorbidities affect the underlying disorder? Br J Pharmacol. 2013;168(7):1531-54.

7. LaFrance WC Jr, Reuber M, Goldstein LH. Management of psychogenic nonepileptic seizures. Epilepsia. 2013;54(Suppl 1):53-67.

8. Moride Y, Abenhaim L. Evidence of the depletion of susceptibles effect in non-experimental pharmacoepidemiologic research. J Clin Epidemiol. 1994;47(7):731-7.

9. National Institute for Health and Care Excellence. Dementia: supporting people with dementia and their carers in health and social care. NICE 2015. http://www.nice.org.uk/guidance/cg42. Accessed 17 Nov 2015.

10. Mourao RJ, Mansur G, Malloy-Diniz LF, Castro CE, Diniz BS. Depressive symptoms increase the risk of progression to dementia in subjects with mild cognitive impairment: systematic review and meta-analysis. Int J Geriatr Psychiatry (Epub 17 Dec 2015).

11. Wilson RS, Boyle PA, Capuano AW, Shah RC, Hoganson GM, Nag S, et al. Late-life depression is not associated with dementiarelated pathology. Neuropsychology. (Epub 3 Aug 2015).

12. Mendez M, Lim G. Seizures in elderly patients with dementia: epidemiology and management. Drugs Aging. 2003;20(11): 791-803.

13. Stewart R, Hotopf M, Dewey M, Ballard C, Bisla J, Calem M, et al. Current prevalence of dementia, depression and behavioural problems in the older adult care home sector: the South East London Care Home Survey. Age Ageing. 2014;43(4):562-7.

14. Care Quality Commission. The state of health care and adult social care in England 2014/15. Available at: http://www.cqc.org. uk/sites/default/files/20151103_state_of_care_web_accessible_4. pdf. Accessed 30 Dec 2015.

15. Mantel N. Synthetic retrospective studies and related topics. Biometrics. 1973;29(3):479-86.

16. Breslow NE, Day NE, Halvorsen KT, Prentice RL, Sabai C. Estimation of multiple relative risk functions in matched casecontrol studies. Am J Epidemiol. 1978;108(4):299-307.

17. Lubin JH, Gail MH. Biased selection of controls for case-control analyses of cohort studies. Biometrics. 1984;40(1):63-75. 